\title{
Antibacterial effect and shear bond strength of an orthodontic adhesive cement containing Galla chinensis extract
}

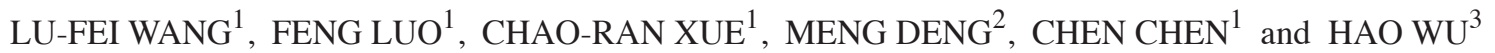 \\ ${ }^{1}$ State Key Laboratory of Oral Diseases, West China Hospital of Stomatology, Sichuan University, Chengdu 610072, P.R. China; \\ ${ }^{2}$ School of Dentistry, University of North Carolina at Chapel Hill, Chapel Hill, NC 27516, USA; ${ }^{3}$ Oral Department, \\ Sichuan Academy of Medical Sciences and Sichuan Provincial People's Hospital, Chengdu 610072, P.R. China
}

Received July 27, 2015; Accepted February 3, 2016

DOI: $10.3892 /$ br.2016.603

\begin{abstract}
Galla chinensis extract (GCE), a naturally-derived agent, has a significant inhibitory effect on cariogenic bacteria. The present study aims to evaluate the antibacterial effect and shear bond strength of an orthodontic adhesive cement containing GCE. A resin-modified glass ionomer cement incorporated GCE at five mass fractions $(0,0.1,0.2,0.4$, and $0.8 \%$ ) to prepare GCE-containing cement for analysis. For the agar diffusion test, cement specimens were placed on agar disk inoculated with Streptococcus mutans (strain ATCC 25175). Following $48 \mathrm{~h}$ incubation, the inhibition halo diameter was measured. To assess bacteria colonization susceptibility, S. mutans adhesion to cement specimens was detected by scanning electron microscopy (SEM) following $48 \mathrm{~h}$ incubation. To evaluate bond strength, a total of 50 metal brackets were bonded on premolar surfaces by using cement (10 teeth/group). Following immersion in an artificial saliva for 3 days, shear bond strength (SBS) was measured. The results demonstrated that GCE-containing samples exhibited a larger bacterial inhibition halo than control, and the inhibition zone increased as the GCE mass fraction increased. SEM analysis demonstrated that $S$. mutans presented a weaker adherent capacity to all GCE-containing cements compared with control, but the difference between each GCE-containing group was not significant. SBS values of each GCE-containing group exhibited no difference compared with the control. In conclusion, GCE-containing adhesive cement exhibits a promising inhibitory effect on $S$. mutans growth and adhesion. Without compromising bond strength, adding GCE in adhesive cement may be an attractive option for preventing white spot lesions during orthodontic treatment.
\end{abstract}

Correspondence to: Dr Hao Wu, Oral Department, Sichuan Academy of Medical Sciences and Sichuan Provincial People's Hospital, No. 32 West Second Section First Ring Road, Chengdu 610072, P.R. China

E-mail: stersewhite@163.com

Key words: white spot lesions, Galla chinensis extract, adhesive cement, antibacterial effect, bond strength

\section{Introduction}

The process of enamel demineralization and the development of white spot lesions around orthodontic brackets, fixed during orthodontic treatment constitutes a serious health issue, with a morbidity rate of $\leq 95 \%(1,2)$. The bonding of orthodontic brackets on the enamel surface can promote dental plaque accumulation, which results in the increased level of cariogenic bacteria, particularly Streptococcus mutans. Demineralization occurs when bacteria metabolize carbohydrates and produce organic acids, dissolving the calcium phosphate mineral of tooth structures (3). Cavitated lesions then develop if no effective therapeutic option is used (4). As the incidence of white spot lesions is increased following orthodontic treatment $(1,5)$, the development of an effective preventive strategy against enamel demineralization around orthodontic brackets is necessary. Novel orthodontic bonding systems with antibacterial properties would be a promising strategy for preventing enamel demineralization, as cariogenic bacteria colonization and proliferation are the primary pathogenesis of white spot lesions (6-8). Previous research has focused on developing novel antibacterial agents for improving orthodontic bonding systems, for example, fluoride, nano-silver, titanium oxide, chlorhexidine and quaternary ammonium (9-11).

The use of substances derived from natural products has been extremely successful in the discovery of novel medicines (12). Galla chinensis, a traditional Chinese medicine, received much attention in the search of new bioactive agents for anti-caries. The bioactive components of Galla chinensis extract (GCE) can suppress the growth of microcosm biofilms and inhibit lactic acid production (13). Furthermore, previous studies have demonstrated that GCE not only has a significant inhibitory effect on enamel demineralization (14), but also effectively promotes the remineralization of initial enamel carious lesion (15-17). Furthermore, GCE seems to be the only natural product with the potential to regulate the balance of enamel demineralization/remineralization (18).

We hypothesized that GCE could be a useful resource for developing an orthodontic bonding system with antibacterial properties. Therefore, the present study aimed to prepare a GCE-containing adhesive cement for orthodontic use, and to evaluate its antibacterial effects and bonding strength. 


\section{Materials and methods}

Preparation of GCE-containing cement. GCE was distilled as described previously (19). Briefly, Galla chinensis produced in Sichuan province, China was dried at $60^{\circ} \mathrm{C}$ for 3 days, powdered, double extracted with distilled water, and then dissolved in ethanol for filtration and evaporation. Subsequently, the GCE was further fractionated by adsorption chromatography and purified by successive column chromatography. The obtained GCE was dissolved in ethanol and adjusted to $\mathrm{pH}$ 5.5. The GCE was incorporated into the liquid of resin-modified glass ionomer cement (GC Fuji ORTHO ${ }^{\text {TM }}$ LC, GC Corp., Tokyo, Japan) at certain mass fractions, which were pre-set to ensure the mass fraction of GCE in cement mixture (following mixing of the liquid with powder). These values were 0 (control group), $0.1,0.2,0.4$, and $0.8 \%$ respectively. Once the GCE-containing cement sample was required for use, the cement liquid was mixed with the powder at a constant ratio, according to the manufacturer's instructions. Following this, $20 \mathrm{sec}$ of LED light curing was performed (Ortholux, 3 M Unitek, Monrovia, CA, USA) for complete polymerization of the cement.

Bacterial suspensions. As one of the most important cariogenic bacteria, S. mutans (strain 25175; American Type Culture Collection, Manassas, VA, USA) was selected for testing the antimicrobial properties of the GCE-containing cement. The bacterial cells were cultured in Brain Heart Infusion (BHI) broth (BD Diagnostics, Franklin Lakes, NJ, USA) and incubated at $37^{\circ} \mathrm{C}$ in a jar with a microaerophilic atmosphere enriched with 5\% $\mathrm{CO}_{2}$ (Oxoid Campygen, Basingstoke, UK) until reaching the initial stationary phase after $48 \mathrm{~h}$ (Optical density at $650 \mathrm{~nm}$ of 0.30 ), and then measured using a Spectronic 20 (Milton Roy, Houston, TX, USA). The microbial suspension was used to inoculate the agar diffusion test plates and to perform the adhesion assay.

Agar diffusion test. An agar diffusion test was adapted to evaluate antibacterial activity. S. mutans suspensions were inoculated on three plates with $20 \mathrm{ml}$ of BHI agar. Five blocks were loaded on each plate and respective cement was introduced and light-polymerized immediately. The plates were then incubated at $37^{\circ} \mathrm{C}$ for $48 \mathrm{~h}$, followed by the measurement of the inhibition halo diameters using a manual caliper. This was repeated three times, and mean values used for analysis.

Bacteria colonization susceptibility. Six tubes containing $0.5 \mathrm{ml}$ of $S$. mutans suspension were prepared. Cements with five mass fractions as described above, were photopolymerized into blocks respectively and then cut in 1-2 mm size solid cubic particles with a scalpel blade. Three solid particles of each cement were placed in respective tubes in order to evaluate bacteria adhesion to cement specimens. A tube containing a pasteurized inactivated bacterial suspension was used as the negative control, in order to evaluate nonspecific adherence of the microorganism. The tubes were then incubated at $37^{\circ} \mathrm{C}$ for $48 \mathrm{~h}$ in an atmosphere enriched with $5 \% \mathrm{CO}_{2}$. Next, in order to remove not firmly adhered bacteria, all particles were washed three times in sterile culture medium and agitated for $10 \mathrm{sec}$ at 1,400 rpm using an SA8 vortex mixer (Bibby Scientific Ltd., Stone, UK). The

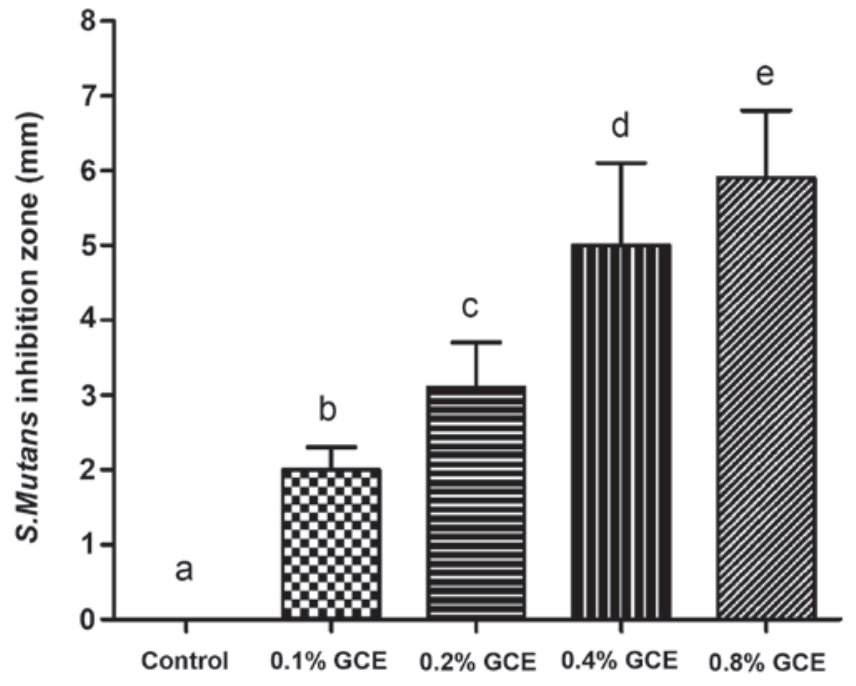

Figure 1. Antibacterial activity of GCE-containing cement in agar diffusion test. GCE-containing samples exhibited larger bacterial inhibition halo than control, and inhibition zone increased as GCE mass fractions increased. Each value is mean $\pm \mathrm{SD}$. Bars with letters indicating values that are significantly different $(\mathrm{P}<0.05)$. GCE, Galla chinensis extract.

specimens were then fixed with glutaraldehyde and osmium tetroxide (both from Sigma-Aldrich, St. Louis, MO, USA), sputter-coated with gold and examined using a Quanta 200 scanning electron microscope (FEI, Hillsboro, OR, USA). Bacteria adhesion was semi-quantitatively evaluated using a 6-level scoring system defined as follows $(20,21): 0$, no bacterial growth; 1 , rare, widely dispersed single cells; 2 , multiple bacterial cells forming a monolayer film; 3 , multiple cells showing active chain-like proliferation with a multilayered film in some areas; 4, a multilayered, homogenous film of bacteria, with the underlying surface visible in some areas, and 5, multilayered, mature colonies of bacterial cells with a spongiform structure and no visible underlying surface.

Bond strength. A total of fifty freshly extracted human premolars collected from orthodontic patients were stored in a $10 \%$ formalin solution at room temperature. Prior to the experiment, the teeth were removed from the preservation solution, washed completely in distilled water and then etched with $37 \%$ phosphoric acid gel at buccal side. All fifty premolars were randomly divided into 5 groups (10 teeth per group). Fifty metal brackets (Gemini, 3M Unitek, Monrovia, USA) were bonded to etched enamel surfaces using five groups of GCE-containing cement respectively. Following light polymerization, the samples were immerged into in artificial saliva (purchased from Canspec Scientific Instruments, Shanghai, China) for three days to simulate intra-oral conditions. A universal testing machine (MTS, Eden Prairie, USA) was used to detect shear bond strength (SBS). The surface area of bracket base was measured, and the breaking load $(\mathrm{N})$ was recorded when bracket detached in the shear mode at a crosshead speed of $0.5 \mathrm{~mm} / \mathrm{min}$. Bond strength was calculated as follows: Bond strength $(\mathrm{MPa})=$ Breaking load $(\mathrm{N}) /$ Area of bracket base $\left(\mathrm{mm}^{2}\right)$.

Statistical analysis. Data were presented as mean \pm standard deviation. One-way ANOVA with post hoc analysis using least significant difference method was performed. All statistical 
A
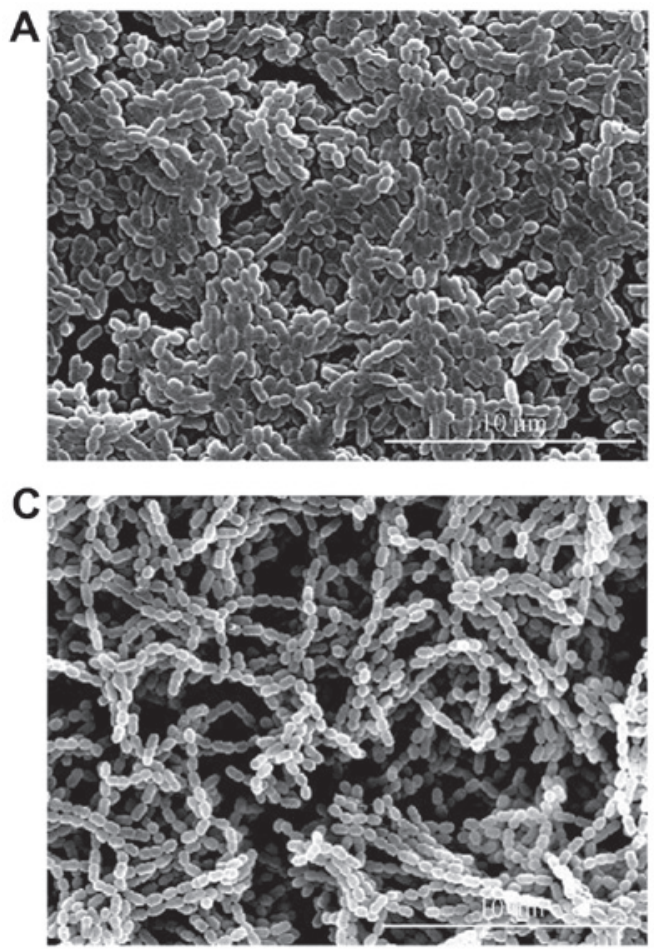

E

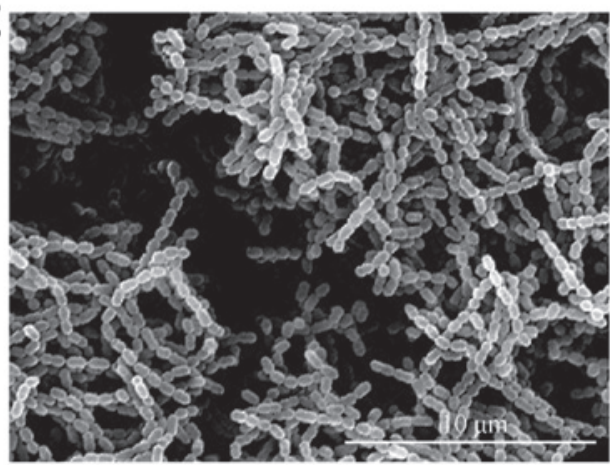

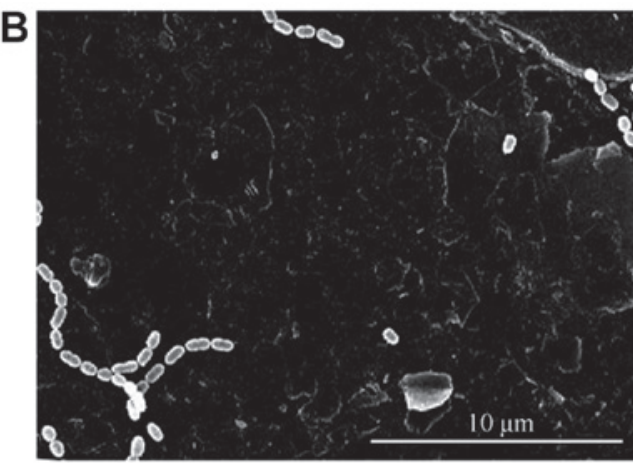
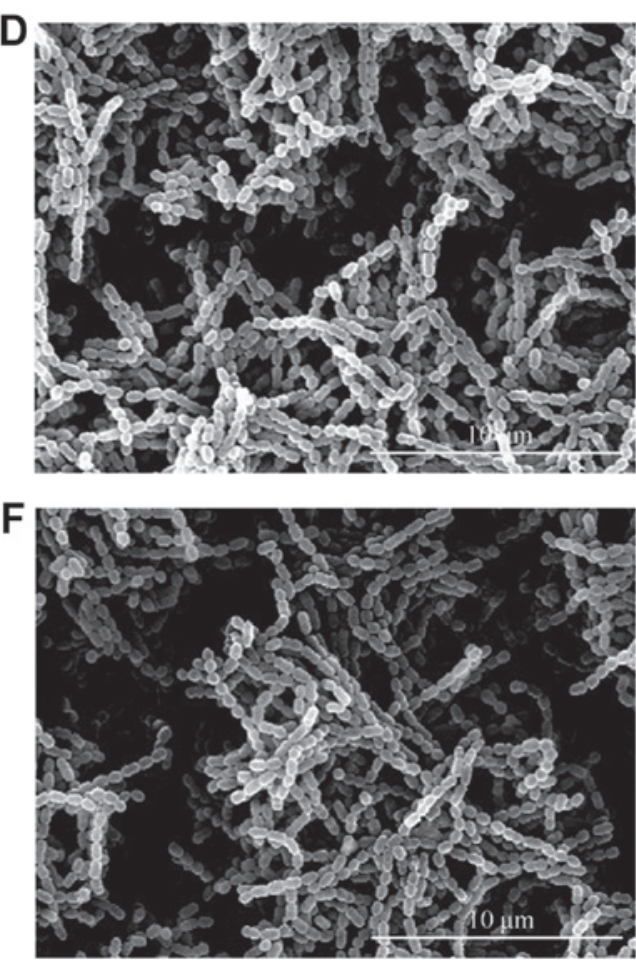

Figure 2. SEM evaluation for $S$. mutans adherence on cement specimens $(20 \mathrm{kV}$, magnification $\times 5,000)$. (A) Control, (B) Inactive $S$. mutans adherence, (C) $0.1 \%$ GCE, (D) $0.2 \%$ GCE, (E) $0.4 \%$ GCE, (F) $0.8 \%$ GCE. S. mutans presented a weaker adherent capacity to all GCE-containing cements compared with control, but the difference between each GCE-containing group was not significant. SEM, scanning electron microscopy; GCE, Galla chinensis extract.

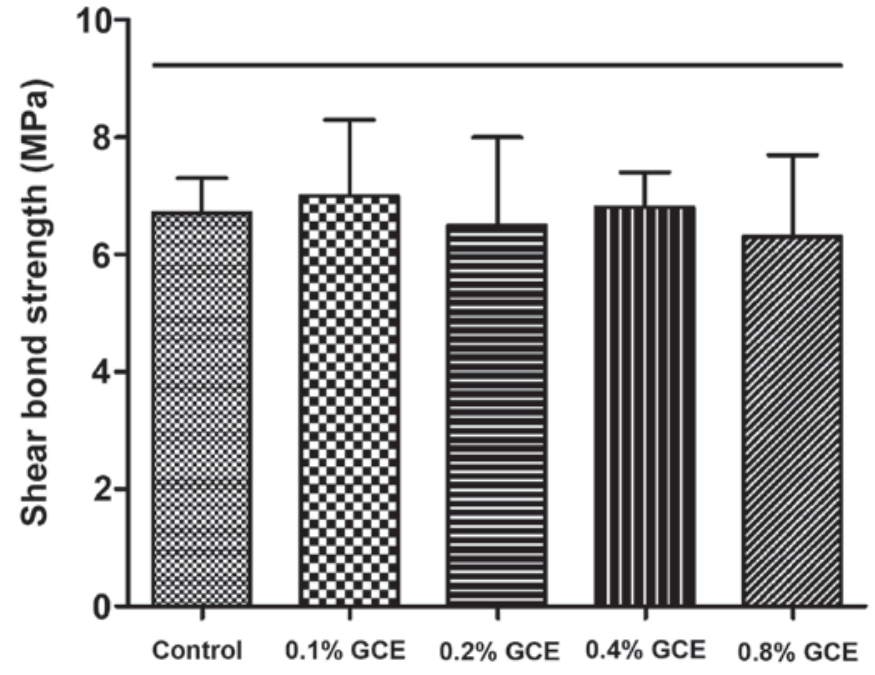

Figure 3. Shear bond strength test. All five groups had shear bond strength that were not significantly different. Each value is mean \pm SD $(n=10)$ Horizontal line indicates all values that are not significantly different from each other $(\mathrm{P}>0.05)$. analyses were performed using the SPSS 17.0 software (SPSS, Inc., Chicago, IL, USA) and $\mathrm{P}<0.05$ was taken to indicate a statistically significant difference.

\section{Results}

Agar diffusion test. As Fig. 1 demonstrates, all GCE-containing samples exhibited an inhibitory effect on $S$. mutans and exhibited larger bacterial inhibition halos than the control $(0 \%$ GCE), with statistical significance $(\mathrm{P}<0.05)$. When the GCE mass fraction increased, bacterial inhibition zone presented a dose-dependent increase. In the present study, the $0.8 \%$ GCE group had the largest diameter of inhibition halo $(5.9 \pm 0.9 \mathrm{~mm})$.

SEM evaluation for $S$. mutans adherence to cement specimens. The tube which contained a pasteurized inactivated bacterial suspension had no specific adherence of $S$. mutans (Fig. 2). Based on semi-quantitative analysis, compared with control sample, $S$. mutans had a weaker adherent capacity to all 
GCE-containing cements, however, differences between each GCE-containing group were not significant.

Evaluation of SBS. The SBS value of the control group (0\% GCE) was $6.7 \pm 0.6 \mathrm{MPa}$. Despite the mass fraction of GCE rising from 0.1 to $0.8 \%$, GCE-containing groups did not exhibit weaker SBS compared with control group, indicating no bond strength impairment (Fig. 3).

\section{Discussion}

It has been demonstrated previously that an increased prevalence of cariogenic bacteria such as S. mutans and Lactobacillus species in the dental biofilm around brackets can promote enamel decalcification and the formation of incipient caries in orthodontic patients $(22,23)$. Modified orthodontic bonding material containing antibacterial components is effective at preventing white spot lesions around brackets $(24,25)$. In general, incorporated components should have a strong ability of inhibiting cariogenic bacteria growth and colonization, and the ability of promoting enamel remineralization is preferred.

In the present study, GCE, a promising anti-caries agent, was used for modifying orthodontic bonding material. GCE has a wide range of biological properties, including antiviral/antibacterial activity and accelerating blood coagulation (26). GCE can not only inhibit certain cariogenic bacteria growth/adherence/acid production (27), but also has the ability of regulating enamel demineralization/remineralization balance $(16,28)$. Chemical analyses have revealed that GCE consists of several monomeric and polymeric polyphenols (e.g., gallotannin, gallic acid) and some other components (such as carbohydrates and proteins). GCE can be isolated to four subfractions (GCEs-A, B, C and D), however, these are less effective than crude GCE regarding antimicrobial and mineralization effects $(16,27,28)$. Therefore, crude GCE was used in the present study.

In the agar diffusion test, the $0.1,0.2,0.4$ and $0.8 \%$ GCE-containing groups all exhibited a significant $S$. mutans inhibition halo, and the antibacterial effect presented a concentration-dependent enhancement. Similarly, $4 \mathrm{mg} / \mathrm{ml}$ GCE was demonstrated to significantly inhibit the growth and metabolism of oral biofilms in previous reports $(13,27)$. Although S. mutans had a weaker adhesion to all GCE-containing cements compared with the control, it appeared that $S$. mutans adherent capacity not be affected by the concentration of GCE, which requires further verification.

SBS test results revealed that adding GCE did not reduce the bond strength of adhesive cement. SBS values of all GCE-containing groups were within adequate range for orthodontic bonding (5.9-7.8 MPa), suggested previously $(29,30)$, indicating that modified adhesive cement containing 0.1-0.8\% GCE meets the required clinical bracket bonding.

Compared with other natural products, the antibacterial activity of GCE appears unremarkable. However, it is so far the only natural product able to regulate enamel demineralization/remineralization balance, which makes it important among various anti-caries natural products (18). The present study discussed the antibacterial property of GCE-containing cement. Based on current findings, it is likely that its effect in preventing enamel demineralization or promoting remineralization would be even more significant. The optimal concentration of incorporated $\mathrm{GCE}$ requires further investigation.

In conclusion, GCE-containing adhesive cement exhibits a promising inhibitory effect on $S$. mutans growth and adhesion. Without impairing bond strength, adding GCE in adhesive cement may be an attractive option for orthodontic bonding.

\section{References}

1. Lovrov S, Hertrich K and Hirschfelder U: Enamel demineralization during fixed orthodontic treatment-incidence and correlation to various oral-hygiene parameters. J Orofac Orthop 68: 353-363, 2007.

2. Richter AE, Arruda AO, Peters MC and Sohn W: Incidence of caries lesions among patients treated with comprehensive orthodontics. Am J Orthod Dentofacial Orthop 139: 657-664, 2011.

3. Brown WE, Gregory TM and Chow LC: Effects of fluoride on enamel solubility and cariostasis. Caries Res 11 (Suppl 1): S118-S141, 1977.

4. Al Mulla AH, Al Kharsa S, Kjellberg H and Birkhed D: Caries risk profiles in orthodontic patients at follow-up using cariogram. Angle Orthod 79: 323-330, 2009.

5. Gorelick L, Geiger AM and Gwinnett AJ: Incidence of white spot formation after bonding and banding. Am J Orthod 81: 93-98, 1982.

6. Spencer CG, Campbell PM, Buschang PH, Cai J and Honeyman AL: Antimicrobial effects of zinc oxide in an orthodontic bonding agent. Angle Orthod 79: 317-322, 2009.

7. Scherer W, Cooper H and Antonelli J: Antimicrobial properties of dental dentin-enamel adhesives. J Esthet Dent 2: 140-141, 1990.

8. Chambers C, Stewart S, Su B, Sandy J and Ireland A: Prevention and treatment of demineralisation during fixed appliance therapy: A review of current methods and future applications. $\mathrm{Br}$ Dent J 215: 505-511, 2013.

9. Passariello C, Sannino G, Petti S and Gigola P: Intensity and duration of in-vitro antibacterial activity of different adhesives used in orthodontics. Eur J Oral Sci 122: 154-160, 2014.

10. Poosti M, Ramazanzadeh B, Zebarjad M, Javadzadeh P, Naderinasab M and Shakeri MT: Shear bond strength and antibacterial effects of orthodontic composite containing $\mathrm{TiO} 2$ nanoparticles. Eur J Orthod 35: 676-679, 2013.

11. Melo MA, Wu J, Weir MD and Xu HH: Novel antibacterial orthodontic cement containing quaternary ammonium monomer dimethylaminododecyl methacrylate. J Dent 42: 1193-1201, 2014.

12. Harvey A: Strategies for discovering drugs from previously unexplored natural products. Drug Discov Today 5: 294-300, 2000.

13. Cheng L, Exterkate RA, Zhou X, Li J and ten Cate JM: Effect of Galla chinensis on growth and metabolism of microcosm biofilms. Caries Res 45: 87-92, 2011.

14. Zhang L, Xue J, Li J, Zou L, Hao Y, Zhou X and Li W: Effects of Galla chinensis on inhibition of demineralization of regular bovine enamel or enamel disposed of organic matrix. Arch Oral Biol 54: 817-822, 2009.

15. Cheng L, Li J, Hao Y and Zhou X: Effect of compounds of Galla chinensis and their combined effects with fluoride on remineralization of initial enamel lesion in vitro. J Dent 36: 369-373, 2008.

16. Chu JP, Li JY, Hao YQ and Zhou XD: Effect of compounds of Galla chinensis on remineralisation of initial enamel carious lesions in vitro. J Dent 35: 383-387, 2007.

17. Cheng L, Li J, Hao Y and Zhou X: Effect of compounds of Galla chinensis on remineralization of enamel surface in vitro. Arch Oral Biol 55: 435-440, 2010.

18. Djakpo O and Yao W: Rhus chinensis and Gallachinensis-folklore to modern evidence: Review. Phytother Res 24: 1739-1747, 2010.

19. Cheng L, Li JY, Huang S and Zhou XD: Effect of Galla chinensis on enhancing remineralization of enamel crystals. Biomed Mater 4: 034103, 2009.

20. Martins Júnior W, De Rossi A, Samih Georges Abi Rached R and Rossi MA: A scanning electron microscopy study of diseased root surfaces conditioned with EDTA gel plus Cetavlon after scaling and root planing. J Electron Microsc (Tokyo) 60: 167-175, 2011.

21. Brambilla E, Ionescu A, Fadini L, Mazzoni A, Imazato S, Pashley D, Breschi L and Gagliani M: Influence of MDPB-containing primer on Streptococcus mutans biofilm formation in simulated Class I restorations. J Adhes Dent 15: 431-438, 2013. 
22. Forsberg CM, Brattström V, Malmberg E and Nord CE: Ligature wires and elastomeric rings: Two methods of ligation, and their association with microbial colonization of Streptococcus mutans and lactobacilli. Eur J Orthod 13: 416-420, 1991.

23. Rosenbloom RG and Tinanoff N: Salivary Streptococcus mutans levels in patients before, during and after orthodontic treatment. Am J Orthod Dentofacial Orthop 100: 35-37, 1991.

24. Brown ML, Davis HB, Tufekci E, Crowe JJ, Covell DA and Mitchell JC: Ion release from a novel orthodontic resin bonding agent for the reduction and/or prevention of white spot lesions. An in vitro study. Angle Orthod 81: 1014-1020, 2011.

25. Patil N, Jawale B, Redasani R, Chaudhari L, Garde JB and Chauhan VS: In vitro caries-preventive effect of fluoridated orthodontic resins against cariogenic challenge stimulation. J Contemp Dent Pract 13: 452-455, 2012.
26. Wu-Yuan CD, Chen CY and Wu RT: Gallotannins inhibit growth, water-insoluble glucan synthesis and aggregation of mutans streptococci. J Dent Res 67: 51-55, 1988

27. Xie Q, Li J and Zhou X: Anticaries effect of compounds extracted from Galla chinensis in a multispecies biofilm model. Oral Microbiol Immunol 23: 459-465, 2008.

28. Zou L, Zhang L, Li J, Hao Y, Cheng L, Li W and Zhou X: Effect of Galla chinensis extract and chemical fractions on demineralization of bovine enamel in vitro. J Dent 36: 999-1004, 2008.

29. Reynolds IR and von Fraunhofer JA: Direct bonding of orthodontic attachments to teeth: The relation of adhesive bond strength to gauze mesh size. Br J Orthod 3: 91-95, 1976.

30. Lopez JI: Retentive shear strengths of various bonding attachment bases. Am J Orthod 77: 669-678, 1980. 